\title{
Derivation of the Planck and Fine-Structure Constant from Assis's Gravity Model
}

\author{
M. Tajmar* \\ Institute of Aerospace Engineering, Technische Universität Dresden, Germany
}

(Dated: April 22, 2015)

\begin{abstract}
Presently, Planck's constant is a fundamental constant that can not be derived from other constants. Assis developed a model based on an extended Weber-type potential energy, that allows calculating gravitational-type forces from neutral oscillating electric dipoles. Here we show that the maximum possible point-mass in his model equals the Planck mass which allows us to derive Planck's constant and the fine-structure constant. We match the exact order of magnitude only requiring a pre-factor that is present in all Weber-type theories and has to be determined empirically. This classical model allows to link electromagnetic, gravitational and quantum properties with one approach.
\end{abstract}

Keywords: Planck Constant, Fine-Structure Constant, Quantum Gravity, Unified Field Theory, Weber Electrodynamics

\section{INTRODUCTION}

The Planck constant $h$ is the pillar of quantum theory being the quantum of action. It describes the proportional constant between the energy and frequency of an atomic oscillator. So far it is listed as one of nature's fundamental constants that can not be derived or calculated from other constants. Only by looking at the fine structure constant, which defines the coupling strength of the electromagnetic interaction between elementary particles and is given by

$$
\alpha=\frac{1}{4 \pi \epsilon_{0}} \cdot \frac{e^{2}}{\hbar c} \approx \frac{1}{137.036},
$$

we can guess that the Planck constant may depend on the electric constant $\epsilon_{0}$, the electric charge $e$ and the speed of light $c$. However, the numerical prefactor has to be determined empirically as one of about 20 empirical parameters in the standard model.

Many attempts have been made to find a viable analytical formula to derive the fine-structure constant using quantum electrodynamics (QED), algebraic identities as well as conjectures based on classical and quantum field theories as recently reviewed by Jentschura and Nándori [1] without convincing success. Planck introduced the quantum of action as an adhoc assumption in order to correctly predict the black-body radiation. As of today, this assumption is called quantum hypothesis as it can not be derived out of theory.

We will show that such a minimum energy condition appears naturally if we use Weber's electrodynamics and a gravitational model that derives gravity from electromagnetism using the Weber force (listed as one of the candidate theories by Jentschura and Nándori [1]). We can then calculate Planck's as well as the fine structure constant only using electromagnetism.

*martin.tajmar@tu-dresden.de

\section{GRAVITATIONAL MODEL AND WEBER'S ELECTRODYNAMICS}

Many years before Maxwell, Wilhelm Weber proposed a single force law that covered all known electromagnetic forces such as the Coulomb and Lorentz force as well as Faraday's induction law [2]. The Weber force between two charges $q_{1}$ and $q_{2}$ separated by the distance $r$ is obtained by the divergence of the Weber potential energy given as

$$
U=\frac{q_{1} q_{2}}{4 \pi \epsilon_{0}} \frac{1}{r}\left(1-\frac{\dot{r}^{2}}{2 c^{2}}\right)
$$

It is possible to derive Maxwell's equations from this potential energy [2] and contrary to Maxwell, Weber's electrodynamics do conserve momentum and angular momentum. However, it also predicts additional properties such as a contribution to inertial mass for charged particles inside a stationary charged spherical shell [3], which has been tested without fully conclusive results [4-7]. Therefore, so far Weber's electrodynamics is only considered a curiosity complimentary to Maxwell at best. It should be noted that a Weber-like force was also successfully proposed for gravity including inertial and frame-dragging effects. Even Schrödinger proposed a similar potential energy as Equ. 2 to study gravitational phenomena and the origin of inertia in rotating reference frames $[8,9]$.

Assis proposed an extension to the Weber energy that allowed him to derive gravitational and intertial forces by using only electromagnetic forces [10-12]. His model is based on the following two assumptions:

1. Assis assumes, that each mass is actually composed of a neutral electric dipole. Each dipole consists of opposing charges $\pm q$ which are separated by a distance $A$ and oscillate with respect to each other at an angular frequency $\omega$. In his simple model, one charge is assumed stationary and the other is oscillating around it. This can be interpreted as a form of string theory where opposite charges are placed on the end of the string.

2. He then proposes a series-expansion of the Weber energy from Equ. 2 to obtain a more general form given by 


$$
U=\frac{q_{1} q_{2}}{4 \pi \epsilon_{0}} \frac{1}{r}\left[1-\alpha\left(\frac{\dot{r}}{c}\right)^{2}-\beta\left(\frac{\dot{r}}{c}\right)^{4}-\gamma\left(\frac{\dot{r}}{c}\right)^{6}-\ldots\right]
$$

where the first coefficient is known as $\alpha=0.5$. The other coefficients should also be on the order of unity but their precise value is not known so far.

Next, the average force between two dipoles is computed by performing an average between all four charges $\left(q_{1 \pm, 2 \pm}\right)$, oscillations in all three axes and a time average over one oscillation perdiod using the derivative of the extended Weber energy. Assis then finds

$$
F=-\frac{7 \beta}{18} \frac{q_{1+} q_{2+}}{4 \pi \epsilon_{0}} \frac{1}{r^{2}} \frac{A_{1-}{ }^{2} \omega_{1}{ }^{2} A_{2-}{ }^{2} \omega_{2}{ }^{2}}{c^{4}} \times\left(1+\frac{\gamma}{\beta} \frac{45 \dot{r}^{2}-18 r \ddot{r}}{7 c^{2}}\right),
$$

where the negative charge is assumed to be stationary with the positive charge oscillating. The same result is found for the opposite case. This equation predicts an attractive force with a $1 / r^{2}$ dependence just like gravity between two electrically neutral oscillating dipoles. A gravitation-like force was therefore derived as a 4th order electromagnetic effect using the extended Weber energy (inertial forces can be interpreted as a 6th order electromagnetic effect). By using elementary charges, atomic distances and nuclear frequencies, one does indeed obtain forces with similar order of magnitude as the produced by two gravitationally interacting atoms. Assis's force has some similarity to the well-known van-der-Waals force between neutral electric dipoles that is also attractive but shows a $1 / r^{6}$ dependence that is different from the behavior of gravitational forces.

In the early stages of the atom model, it was not clear why the atom was a stable configuration with an electron rotating around a nuclear core that should loose energy due to radiation. The principles of quantum mechanics and the waveparticle duality solved this issue. Surprisingly, the approach of mass as a consequence of vibrating charged strings is also a stable configuration as it can be shown due to different reasons. If mass needs at least one vibrating dipole, a single charge itself an the end of the vibrating string is massless. It can be seen as a "charged photon" that similarily moves at the speed of light according to special relativity with a certain vibration frequency that is related to mass.

The properties of such hypothetical massless charges are discussed in the recent literature. A detailed analysis shows that such particles do not radiate $[13,14]$, therefore a vibrating charged string with massless charges is also a stable configuration.

\section{GRAVITATIONAL LIMIT AND THE PLANCK CONSTANT} law,

By equalling the Assis force with Newton's gravitational
$F=-\frac{7 \beta}{72 \pi \epsilon_{0} c^{4} r^{2}} \cdot q_{1} A_{1}^{2} \omega_{1}^{2} \cdot q_{2} A_{2}^{2} \omega_{2}^{2}=-\frac{G}{r^{2}} \cdot m_{1} \cdot m_{2}$,

we can get a general expression of mass as a function of the dipole properties

$$
m=\sqrt{\frac{7 \beta}{72 \pi \epsilon_{0} G}} \cdot \frac{q A^{2} \omega^{2}}{c^{2}}
$$

We can now use this model to calculate the maximum possible point-mass, which is the definition of the Planck-mass, that allows us to calculate $h$. If a particle has the Planck mass, it's Compton wavelength equals the Schwarzschild radius and therefore no larger point-mass is allowed. The maximum point-mass-limit here is based on the following two assumptions

1. At the elementary particle level, the highest charge is the elementary charge $e$.

2. The largest oscillation velocity is limited by the speed of light $c$ as discussed above. The Assis model uses simple 1D oscillations where the charge position is given by $A \cdot \sin (\omega t)$ and his force equation 4 is an average performed over the oscillation period. We will therefore perform a similar calculation and assume that the average absolute velocity over the oscillation period is limited by the speed of light. We then get

$$
|\bar{v}|=\frac{A \omega}{\pi}=c
$$

We now put these limits into the mass equation 6 and equal it to the Planck mass,

$$
m_{P}=\sqrt{\frac{7 \pi^{3} \beta}{72 \epsilon_{0} G}} \cdot e=\sqrt{\frac{\hbar c}{G}}
$$

which allows us to express the reduced Planck constant as

$$
\hbar=\frac{h}{2 \pi}=\frac{7 \pi^{3} e^{2} \beta}{72 c \epsilon_{0}}=2.92 \times 10^{-35} \cdot \beta
$$

This is exactly the right order of magnitude that we expect and we obtain the right value with $\beta=3.62$. From Weber's and Assis's approach we only knew that $\beta$ should be on the order of unity - which it is. Moreover, Assis developed a simple 1D oscillation model so further improvements might lead to a slightly different value for $\beta$. We can see clearly how the Planck constant can be extracted from the same constants that are present in the fine-structure constant equation 1.

We can use this result to also get a simple expression for the fine-structure constant and obtain 


$$
\alpha=\frac{e^{2}}{2 c \epsilon_{0} h}=\frac{18}{7 \pi^{4} \beta}=\frac{1}{37.88 \cdot \beta}
$$

The famous energy-mass equivalence can also naturally be extracted from the vibrating string-model. The rotational energy of the dipole is related to the mass, amplitude and frequency of the dipole-string. We also need to account for the degrees of freedom similar to a diatomic gas. Using the speed of light limit from Equ. 7, we can then express

$$
E=\frac{7}{2} \frac{I}{2} \omega^{2}=\frac{7}{4} \frac{m A^{2}}{4} \omega^{2}=\frac{7 \pi}{16} m c^{2} \approx m c^{2}
$$

Assis showed that the Weber energy can describe all electromagnetic phenomena. Schrödinger and Assis used the same Weber-type potential energy to derive gravitational forces including frame dragging effects. As we could show here, the extended Weber potential can not only describe electromagnetic and gravitational forces, but that it also includes the Planck constant or a minimum action. This may open up an alternative approach to study links between quantum theory and gravitation. Of course we used a classical approach and a simple model that will need further refinements in the future, however, the mass model as a one-dimensional oscillating electric dipole-string could be an interesting alternative or complementary to present Higgs- and string theory approaches.

\section{ACKNOWLEDGEMENT}

I would like to thank A. Assis for many discussions and comments on his gravitational model.
[1] U. Jentschura and I. Nándori, Eur. Phys. J. H 39, 591613 (2014).

[2] A. Assis, Weber's Electrodynamics (Kluwer Academic Publishers, Dordrecht, 1994).

[3] A. Assis, J. Phys. Soc. Jpn. 61, 1418 (1993).

[4] V. Mikhailov, Ann. Fond. Louis de Broglie 24, 161 (1999).

[5] V. Mikhailov, Ann. Fond. Louis de Broglie 26, 633 (2001).

[6] V. Mikhailov, Ann. Fond. Louis de Broglie 28, 231 (2003).

[7] J. Junginger and Z. Popovic, Can. J. Phys. 82, 731 (2004).

[8] E. Schrödinger, Ann. d. Phys. 382, 325 (1925).

[9] E. Schrödinger, in Machs Principle From Newtons Bucket to Quantum Gravity, edited by J. B. Barbour and H. Pfister
(Birkhäuser, 1995) pp. 147-158.

[10] A. Assis, Can. J. Phys. 70, 330 (1992).

[11] A. Assis, in Advanced Electromagnetism: Foundations, Theory and Applications, edited by T. W. Barrett and D. M. Grimes (World Scientific, Singapore, 1995) pp. 314-331.

[12] A. Assis, Ann. Fond. Louis de Broglie 27, 149 (2002).

[13] B. Kosyakov, J. Phys. A 41, 465401 (2008).

[14] K. Lechner, (2014), arXiv:1405.4805v1 [hep-th]. 